\title{
Le soupçon
}

The Suspicion

\section{Christine Henry}

\section{(2) OpenEdition}

Journals

Édition électronique

URL : http://journals.openedition.org/span/1099

DOI : $10.4000 /$ span. 1099

ISSN : 2268-1558

\section{Éditeur}

École pratique des hautes études. Sciences humaines

\section{Édition imprimée}

Date de publication : 1 novembre 1989

Pagination : $1-10$

ISSN : 0294-7080

\section{Référence électronique}

Christine Henry, "Le soupçon », Systèmes de pensée en Afrique noire [En ligne], 9 | 1989, mis en ligne le 15 octobre 2013, consulté le 21 avril 2019. URL : http://journals.openedition.org/span/1099 ; DOI : 10.4000/span.1099 


\section{LE SOUPÇON}

par

\section{Christine Henry}

Il y a deux jours, alors que le village était assoupi sous la chaleur, à l'heure où les hommes sont en forêt et où les femmes, délaissant les gros travaux, nouent des jupes de fibre à l'ombre des vérandas, des cris stridents retentirent. Deux hommes, portant sur une civière un cadavre enroulé dans une natte, venaient d'entrer au village. La défunte fut déposée sous la véranda du grenier d'un homme de son clan.

Fatou était morte ${ }^{2}$.

En un instant tout ce qui vivait au village s'agglutina autour du corps. De cette mêlée s'élevaient pleurs, lamentations, hurlements. Ce chaos qui semblait l'expression spontanée d'un immense chagrin s'organisa bientôt. Les femmes couraient de la morte à la maison, revenaient avec les accessoires qui servent à leurs cérémonies (coiffes de bois, éventails de plumes), et les agitaient au dessus du corps ${ }^{3}$. Un tambour fut apporté, les pleurs se firent chant.

IIl s'agit d'Inorei, village de Carhabaque, île à l'est de l'archipel des Bijagos (GuinéeBissau).

${ }^{2}$ Agée d'une trentaine d'années, la jeune ferme était malade depuis trois ans. Dernièrenent, elle était allée consulter des guérisseurs au sud du continent, c'est là qu'elle était morte.

3 Fatuu était l'une des deux responsables du groupe apuduta chargé du rituel nubir kusina, "honorer les ainés". Ce cycle rituel est suivi par les homes et les femmes entre 20 et 35 dans des promotions parallèles.

Systèmes de pensée en Afrique Noire, 9, 1989 
Quelques femmes de la promotion de Fatou se chargèrent d'aller avertir les villages voisins. Elles s'éloignèrent sur le chemin, chantaient et dansaient en battant tambour. Pendant ce temps, d'autres préparaient la morte. Le corps fut sommairement lavé, puis assis dans un fauteuil de toile pliant qu'un voisin avait prêté pour la circonstance. Des tee-shirts, les siens et ceux que les villageois commençaient à offrir, lui furent enfilés les uns par dessus les autres. Pour ce faire, il fallait à chaque fois dénouer le foulard jaune vif qui, passé sous le menton, maintenaient les mâchoires fermées et gardait sa tête appuyée contre un poteau de la véranda. A chaque fois, il fallait réajuster son mouchoir de tête et lui reposer sur le nez ses lunettes de soleil. Le bas du corps était enveloppé dans une couverture de couleur vive qui cachait ses jupes et ses pieds chaussés de sandales de plastique ${ }^{4}$.

Des femmes des villages voisins venaient grossir la masse de celles qui, assises par terre en face du cadavre, pleuraient et psalmodiaient des bribes de chants cérémoniels féminins au rythme monotone du tambour. D'autres, debout, ébauchaient un pas de danse ou se balançaient sur place, les bras à demi-levés, les mains ouvertes vers le ciel. Les proches de la morte, accroupies à ses pieds, agrippaient ses mains, ses jambes, dérangeant le fragile équilibre du corps. Parfois, suffoquant de pleurs, elles se levaient et passaient derrière le grenier pour pousser un hurlement sinistre.

De retour au village, les hommes âgés s'étaient regroupés à l'ombre d'une maison voisine; les plus jeunes assistaient le mari de la morte qui, dans l'obscurité de sa chambre, poussait des cris

\footnotetext{
4 Ordinairement, les fermes vont nu-pieds et sont vêtues de deux ou trois jupes de fibre posées sur les hanches leur arrivant à mi-cuisse. Elles aiment à porter des tee-shirts, qui sont le plus souvent en haillons. Lors des cérémonies nubir kusina, elles superposent quatre ou cinq jupes de fibre très épaisses et très longues et portent des tee-shirts en bon état, de couleur vive. Elles se parent avec des foulards drapés sur la tête et noués en flots au bras, mettent des socquettes, des sandales en plastique, des lunettes de soleil et s'arment de gros parapluies noirs ou de tubes néon. L'habillement de Fatou était un mélange de ces deux modèles. Elle portait des jupes courtes (vie quotidienne) mais on la parait des accessoires des moments rituels (tee-shirts, chaussures, lunettes).
} 
lamentables. D'autres avaient commencé à creuser la tombe dans la maison de Batipon, un frère de Fatous.

Toute activité sans rapport avec l'enterrement avait cessé dans le village, et ceux que l'on voyait passer ça et là allaient fouiller au fond d'une malle à la recherche d'un cadeau à offrir : jupe de fibre, foulard, pagne, tabac, argent, alcool. Près du corps les lamentations continuaient, prenaient parfois des allures de fête quand les vocalises des femmes répondaient à un coup de fusil ou saluaient un cadeau particulièrement fastueux. Du riz en grain et en épi était jeté, prestement ramassé par une voisine ou picoré par les poules. En fin d'après-midi, le corps, enroulé dans une couverture, fut rapidement porté dans la maison où avait été ouverte la tombe. Il fut enfoui avec les quelques objets que possédait la morte et quelques uns des cadeaux. Les autres offrandes réparties entre les proches parentes utérines regagnèrent l'abri des malles au fond des greniers. Seules les jupes de fibre furent pendues à un bâton posé horizontalement sur deux bois fourchus près de la maison du deuil. Un paroxysme de cris et de pleurs marqua le déplacement et l'ensevelissement.

Les parentes et les femmes venues des villages voisins se préparèrent à dormir dans la maison. Longtemps la nuit bruissa du murmure des hommes restés à veiller et des chants plaintifs des femmes. Les aînées se réunirent dans le sanctuaire et décidèrent que le lendemain il serait procédé à l'interrogation de la morte. Cette décision n'allait pas sans entraîner des soucis pour le village car en raison de l'importance de la défunte il faudrait battre le tambour de bois et donc faire venir le tambourinaire d'Indena ${ }^{B}$.

5 Quel que soit le statut du mort, ce sont les nalo, les jeunes gens non initiés, qui creusent la tombe, un trou profond au fond duquel est ouverte une galerie horizontale, n'importe uà dans la maison, sans autre souci que de ne pas creuser là où déjà repose un corps. Les nalo transportent le cadavre, l'enfouissent et comblent la tombe. Ils sont seuls à pénétrer dans la maison au moment de l'ensevelissement. Quand ils ont terminé, ils sortent en file indienne et quittent le village pour aller se laver au puits.

5 Indena est à deux heures de marche au sud d'Inorei ; les deux villages sont en rivalité et entretiennent des relations agonistiques. Inorei a bien un tambourinaire mais il ne sait pas ou ne sait plus jouer. Certains disent qu'il a été mal choisi et n'a jamais su jover, d'autres disent qu'il a perdu son savoir à la suite d'évènements obscurs. 
Le lendemain, l'activité du village se concentra à nouveau autour de la maison du deuil : les vieilles femmes assises en groupe devant la maison continuaient à pleurer, les autres allaient et venaient avec des plats. Quelques hommes commencèrent à fabriquer la civière qui allait servir à l'interrogation. Le joueur de tambour arriva avec son acolyte et ils installèrent l'instrument en face de la maison.

La civière fut achevée : étroite, longue d'un mètre cinquante environ, de bois liés dont certains en forme d'arceaux pour figurer l'emplacement d'un corps. Dans cet espace fut introduite une natte enroulée à l'extrémité de laquelle dépassaient deux fétiches semblables à la paume d'une main prolongée d'une fourche à trois pointes que l'on appelle ulan-koko, "medecine-main". Ces fétiches sont habituellement gardés dans la maison du chef rituel, sur l'autel qui abrite l'orebuko, l'esprit du village". Sur les arceaux on attacha une tige de bananier et sa fleur. L'ensemble fut recouvert d'un pagne rouge à bandes jaunes, plié et attaché au bord de la civière. Les hommes avaient également confectionné deux anneaux de portage en feuilles de bananier et cueilli des feuilles rondes de kañama ${ }^{8}$ qui reposeraient entre l'anneau et la civière sur la tête du premier porteur.

En début d'après-midi, après que le tambour de bois eut appelé tous les villageois à se regrouper, le plus jeune initié du village et un autre homme (seul le premier porteur a une importance) posèrent la civière sur leur tête et se rendirent dans le bas du village, devant la case miniature de l'orebuko akpenena, l'esprit du bas ${ }^{8}$. Quand tous

7 Orebuko, (pl. arebuko), est un terme générique désignant tous les fétiches; il signifie également le principe vital des êtres animés. Cette entité perdure à leur mort, le terme est donc aussi employé dans le sens de "défunt" et désigne les femmes possédées par les morts à certains moments rituels.

- Le kañama est une liane, de la famille des ficus, qui tient une grande place dans la vie bijogo. Ses feuilles sont utilisées, sous diverses formes, dans la thérapie indigène. Près du village, un kañama qui a pris des proportions gigantesques et enlace un jeune fromager est un fétiche féminin important.

' Le village, outre les divisions en quartiers, est divisé en deux moitiés: akpenena et aletana, le haut et le bas. Les âmes des morts, avant d'entreprendre le voyage qui les mène dans l'au-delà, se présentent devant l'esprit du bas. Ce fétiche se compose de cinq "medecine mains" posées sur un plateav qui est gardé dans une petite case ronde. Toutes les nuits, un aîné ou une aînée vient allumer du feu dans cette case. Lorsqu'une cérémonie est adressée à ce fétiche, le plateau portant les mains est sorti de la case et posé devant une toute petite 
les villageois par groupe de sexe et d'âge se furent assis près de la petite case, trois hommes initiés prirent successivement la parole. Ils dirent en substance la même chose. Ils rappelèrent que Fatou avait été longtemps malade et qu'aujourd'hui, elle était morte. Ils demandèrent à l'esprit de les aider à découvrir ce qui avait tué Fatou. Etait-elle morte parce que quelqu'un lui en voulait? C'était cela, qu'eux tous réunis - et l'orateur d'interpeller les personnes les plus importantes du village - c'était cela qu'ils voulaient savoir. Bien des hypothèses avaient été avancées par les guériseurs consultés par Fatou et son frère, mais ce qu'ils voulaient savoir aujourd'hui, c'était la vérité ! Que l'esprit du bas les aide dans leur quête !

Le sacrificateur de l'esprit du bas tua un poulet offert par le frère de Fatou. Il lui coupa la tête, laissa le corps sauter en tous sens et quand l'animal s'arrêta, humecta de son sang les cinq mains de l'esprit. Cela fait, Oringa, l'aîné du clan Onoka Munio, le clan de Fatou, qui dirigeait la cérémonie, donna le signal de se rendre sur la grande place du village. Les mêmes porteurs reprirent la civière et la posèrent devant la tonnelle du chef rituel. Le chef prit place sous la pergola en compagnie de la prêtresse et du chef d'un village voisin. Okati, la femme qui a la charge de porter la civière lors de l'interrogation des morts quand il s'agit de femmes ou d'hommes non initiés, les rejoignit. Les villageois s'assirent autour de la place. Une fillette apporta le fétiche de l'esprit du village, un homme alla chercher le fétiche des hommes initiés et le posa près du premier face à la tonnelle. Le chef se leva et sacrifia successivement trois poulets, le premier offert par le clan du père de Fatou, les autres par le clan de Fatou. Il procéda de la même manière que la première fois. Okati s'était levée et semblait se concentrer; elle mit ses mains dans les mains de la prêtresse puis des chefs et s'approcha de la civière. Une femme se détacha de l'assistance et vint derrière elle. Elles se coiffèrent des anneaux de portage, levèrent la civière et la

maison de bois, qui ressemble à une niche, édifiée en face de la case ronde. Cette maisonnette est encadrée de vieilles bouteilles de gaz rouillées, des culs de bouteilles de verre sont onterrés à son seuil, un mât surmonté d'un panneau où se devine une ancienne inscription, qui est probablement un ancien signal maritime, est fiché à son côté. 
posèrent sur leur tête en la maintenant des deux mains. Elles vacillaient comme si elles avaient porté quelque chose de très lourd. Enkeme, le maître de l'initiation, un des hommes les plus vieux et les plus respectés du village, se leva et se plaça devant la civière. Il dit :

- Fatou, est-ce toi qui est ici dans la civière ?

La civière acquiesça en penchant brusquement vers la droite ${ }^{10}$. Enqueme continua :

- As-tu vu ton père? - Oui.

- Ton père t'a-t-il dit ce qui t'avait tuée ? - Oui.

- Vas-tu nous le dire aujourd'hui ? - Oui.

- Maintenant j'ai assez parlé. Ce sont les tiens qui vont venir te questionner. Tu dois parler sans honte car nous sommes tous rassemblés pour connaître la vérité.

Quand il eut fini de parler, la civière recula doucement, effectua un mouvement tournant et partit en courant jusqu'au sanctuaire où elle entra. Cette manoeuvre déconcerta un moment 1'assemblée; quelques aînées se précipitèrent à sa suite. Après quelques minutes, la civière revint sur la place suivie des vieilles femmes qui jodlaient. Elles se rassirent et la séance reprit. Sesimo, le père ${ }^{11}$ de Fatou, se leva et vint se placer devant la civière.

- Fatou, est-ce toi qui est ici ? - Oui.

- As-tu vu ton père? - Oui.

- T'a-t-il dit ce qui t'avait tuée ? - Oui.

- Est-ce de moi qu'est venu ce qui t'a tuée ? - Non.

10 C'est la première porteuse qui effectue le mouvement, la seconde ne fait que suivre les implusions. Pour répondre "oui", les fermes penchent la civière vers la droite. Pour répondre "non", elles l'agitent dans un tremblement qui évoque le mouvenent de va-et-vient d'une tête secouée en signe de dénégation. A ces mouvements effectués sur place, il peut s'en ajouter d'autres: mouvements affirmatífs de déplacement en avant jusqu'à toucher l'épaule du questionneur, en manière de salutations; mouvements négatifs en arrière comme si la civière était offusquée par la question.

${ }^{11}$ Ego appelle ote, père, son père légal et tous les hormes du même matrilignage. Les droits et devoirs paternels se transmettent dans le matrilignage. Les pères de Fatou dont il est question ici sont son père légal qui est mort, un neveu utérin de cet houme qui est mort également, et le frère de ce dernier, l'actuel pater de Fatou. 
- Est-ce mon frère aîné, le tambourinaire, celui qui a hérité de toi à la mort de ton père, qui t'a tuée ? - Non .

- Je possède un fétiche qui peut tuer les gens, as-tu fait quelque chose qui aurait suscité sa colère ? - Non.

- Je m'arrête ici, d'autres vont venir te poser des questions car nous voulons savoir la vérité.

Oringa, l'aîné du clan des Onoka Munio, se leva.

- Fatou, est-ce toi qui est là ? - Oui.

- As-tu vu ton père? - Oui.

- Ton père t'a-t-il dit ce qui t'avait tuée ? - Oui.

- Dans notre clan, il y a beaucoup de personnes qui sont mortes dont nous n'avons pas fait les levées de deuil. Même moi, qui parle ici, je ne le fais pas car je suis vieux et je n'ai plus de force. Est-ce de cela que tu es morte ? - Non.

- Dans le monde d'aujourd'hui, les gens n'ont plus de respect, ils se moquent les uns des autres. L'un qui passe est pauvre, tu le regardes et tu ris de lui, il te tue pour laver l'offense, est-ce cela qui t'a tuée ?

La civière nia, recula comme un animal impatient et partit en courant vers le sanctuaire. Comme la première fois, les vieilles femmes la suivirent. Quelques instants passèrent, la civière revint.

Oringa reprit :

- J'ai entendu dire qu'un de tes frères avait volé dans la maison d'un Balante ${ }^{12}$, est-ce cet homme qui t'a tuée pour se venger ? - Non.

- Est-ce l'esprit ${ }^{13}$ de notre clan, celui qui peut tuer, qui t'a fait mourir? - Non.

A nouveau, la civière partit en courant vers le sanctuaire. Quand elle revint, Oringa poursuivit :

- A toutes mes questions, tu réponds par la négative. Fatou, est-ce moi qui t'ai tuée? - Non.

12 Ethnie du continent, les Balante ont la réputation d'être voleurs.

${ }^{13}$ Oringa emploie, ici, le terme elamundi pour désigner cette puissance. Chaque clan possède un elamundi mais tous les kolamundi ne sont pas des esprits claniques. Par contre, ils ont tous la même origine, ce sont des pythons qui sont allés vivre dans la mer. 
- J'ai entendu dire que tu étais peut-être morte d'avoir mangé d'un plat du sanctuaire, est-ce de cela que tu es morte ?

La civière acquiesça plusieurs fois avec violence. Oringa reprit la parole :

- Je suis triste et mécontent de tout cela car tu avais fait tout le travail d'honorer les aînés, tu devais finir cette année et tu es morte. Tu ne pourras pas manger les fruits de ton travail, toute ta fatigue n'aura servi à rien.

Il retourna dans l'assemblée et Nimapa, un frère de Fatou, celui qui l'avait accompagnée dans ses quêtes thérapeutiques, se leva et prit sa place.

- Fatou ? - Oui.

- Je ne vais pas recommencer à poser toutes les questions qui ont déjà été posées. Tu as été tuée dans le sanctuaire ? - Oui.

- Vraiment tu es morte là ? - Oui.

- Tout le monde meurt, il n'est personne qui échappe à la mort, mais nous devons savoir ce qui t'a tuée. Quand tu étais malade, nous sommes allés voir bien des guérisseurs, j'ai fait faire beaucoup de divinations. L'un de ces guérisseurs, déjà, avait dit que tu étais morte d'avoir mangé d'un plat du sanctuaire, ainsi véritablement, c'est bien de cela que tu es morte ? - Oui ${ }^{14}$.

Nimapa alla se rasseoir; Mukis, une femme, se leva vivement et se jeta à genoux devant la civière. Cette femme partage avec une autre, Ilisia, ce jour-là absente, la responsabilité, côté Akpenena, du groupe Aragui, groupe hiérarchiquement supérieur à celui de Fatou . - Tu as été tuée dans le sanctuaire ? - Oui.

- Nous avons deux côtés, Akpenena et Aletena, est-ce de notre côté que tu es morte? - Oui.

14 Lorsque les fermes "honorent leurs ainées", l'essentiel de leur travail consiste à cuisiner dans un jot de terre spécial un plat composé de riz, d'huile de palme et de coquillages. Les pots sont apportés au sanctuaire où les responsables de la promotion répartissent la nourriture en plusieurs parts. Une est réservée aux morts, une autre au chef rituel, une autre à la prêtresse. Le reste est mangé par les aînées dans le sanctuaire. Les femmes qui ont cuisiné $n$ 'assistent pas au repas, elles n'ont pas le droit de manger. On dit qu'elles mourraient si, à ce moment-là, elles touchaient à cette nourriture. Elles ne goûteront les plats que le lendemain quand les aînèes répartissent l'excédent dans le village. 
- Est-ce de ma main que tu as reçu la nourriture qui t'a fait mourir?

- Non.

- Mais c'est bien de notre côté que tu es morte ?

Les porteuses penchèrent violemment la civière vers la droite en signe d'acquiescement mais sans achever le mouvement la lâchèrent brusquement et regagnèrent leur place. Mukis se releva et retourna vers les femmes tout en continuant à parler avec une intense émotion. - C'est de notre côté qu'elle est morte, mais tous ici savent que ce n'est pas moi qui l'ai tuée. J'aurais trop honte d'avoir fait une pareille chose. Il est dommage que les femmes ait lâché la civière et que nous ne puissions savoir avec certitude qui l'a tuée !

La cérémonie était finie, l'assemblée se dispersa.

Seule une responsable avait pu prélever de la nourriture réservée et la présenter à Fatou. Puisque la morte avait disculpé Mukis, il ne pouvait s'agir que de l'autre femme : Ilisia. Native d'Inorei, elle n'y habite pas car elle a épousé le chef rituel d'un village du sud de l'île. Elle ne revient à Inorei que pour le temps des cérémonies. Actuellement, elle ne se trouve même pas à Canhabaque car tout son village a migré temporairement sur une petite île du sud pour la saison culturale. A son retour, elle ne pourra ignorer le soupçon qui pèse sur elle, que fera alors la seconde victime de la maladie de Fatou? 